\title{
El consumo de granos se asocia a una disminución del riesgo de mortalidad total y por causa cardiovascular
}

Dietary whole grain intake is associated with reductions in all cause and cardiovascular mortality

Wu H, y col. JAMA Intern Med. 2015;175(3):373-384.

\section{Objetivos}

Establecer la relación entre el consumo de granos integrales y el riesgo de mortalidad total y causa-específica.

\section{Diseño y lugar}

Estudio prospectivo de cohortes. Estados Unidos.

Pacientes

Se tomaron dos cohortes: el Nurses' Health Study (NHS), con seguimiento de 1984 a 2010, y el Professionals Follow-Up Study (PFS), con seguimiento de 1986 a 2010. De las mismas se excluyeron a participantes con cáncer, accidente cerebrovascular, o enfermedad coronaria al inicio del seguimiento; y/o con información nutricional insuficiente y/o inverosímil (por ejemplo, que hayan consumido menos de $500 \mathrm{Kcal}$ diarias por 25 años). La población incluida fue de 74.341 mujeres (NHS) y 43.744 hombres (PFS).

\section{Medición de resultados principales}

Para recabar información nutricional, se tomaron datos al inicio del seguimiento y cada cuatro años a través del cuestionario validado, Food Frequency Questionnaire (FFQ). incluyendo el consumo promedio diario de granos por participante.

Para la información sobre mortalidad se tomo como fuente un familiar del participante, el registro civil o el National Death Index. Luego, se estudiaba el certificado de defunción y la historia clínica del participante clasificando el motivo de muerte de acuerdo al International Classification of Diseases (ICD-8). A través de cuestionarios basales y cada cada años, se estudiaron también datos como el índice de masa corporal (IMC), tabaquismo, consumo de alcohol, actividad física, diabetes, hipertensión arterial, hipercolesterolemia y un puntaje de calidad nutricional de la dieta (sigla en inglés AHEI).

\section{Resultados principales}

Se agrupó a los participantes en quintilos crecientes según la media de consumo de granos y se elaboraron tres modelos que relacionaban dicho consumo con la mortalidad, ajustando por distintas covariables. El riesgo fue expresado en Hazard Ratios (ver Tabla 1).

Tabla 1. Mortalidad total y causa-especifica por quintilos crecientes de consumo diario de granos.

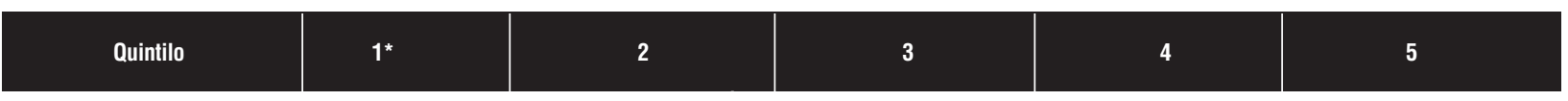

Mortalidad total: Hazard Ratio (IC95\%)

\begin{tabular}{|c|c|c|c|c|c|}
\hline Modelo 1 & 1 & $0,75(0,73$ a 0,78$)$ & $0,65(0,63$ a 0,68$)$ & $0,59(0,57$ a 0,61$)$ & $0,54(0,52$ a 0,56$)$ \\
\hline Modelo 2 & 1 & $0,98(0,95$ a 1,02) & $0,97(0,93$ a 1,01$)$ & $0,95(0,91$ a 0,99$)$ & $0,88(0,85$ a 0,92$)$ \\
\hline Modelo 3 & 1 & $0,99(0,95$ a 1,02) & $0,98(0,95$ a 1,02) & $0,97(0,93$ a 1,01$)$ & $1,04(0,98$ a 1,11$)$ \\
\hline \multicolumn{6}{|c|}{ Mortalidad cardiovascular: Hazard Ratio (IC95\%) } \\
\hline Modelo 1 & 1 & $0,73(0,68$ a 0,78$)$ & $0,63(0,59 a 0,68)$ & $0,54(0,50$ a 0,58$)$ & $0,52(0,48$ a 0,56$)$ \\
\hline Modelo 2 & 1 & $0,94(0,87$ a 1,01$)$ & $0,93(0,87$ a 1,01$)$ & $0,86(0,8$ a 0,94$)$ & $0,83(0,77$ a 0,89$)$ \\
\hline Modelo 3 & 1 & $0,94(0,88$ a 1,01) & $0,94(0,87$ a 1,01$)$ & $0,87(0,8 \mathrm{a} 0,94)$ & $0,85(0,78$ a 0,92$)$ \\
\hline \multicolumn{6}{|c|}{ Mortalidad por cáncer: Hazard Ratio (IC95\%) } \\
\hline Modelo 1 & 1 & $0,81(0,77$ a 0,86$)$ & $0,75(0,7$ a 0,8$)$ & $0,69(0,65$ a 0,73$)$ & $0,63(0,59$ a 0,67$)$ \\
\hline Modelo 2 & 1 & $1,02(0,96$ a 1,08) & $1,05(0,99$ a 1,12$)$ & $1,04(0,97$ a 1,1$)$ & $0,96(0,9$ a 1,03$)$ \\
\hline Modelo 3 & 1 & $1,02(0,96$ a 1,08) & $1,05(0,99$ a 1,12) & $1,04(0,98$ a 1,11) & $0,97(0,91$ a 1,04$)$ \\
\hline
\end{tabular}

Modelo 1: ajustado por edad; Modelo 2: edad, etnia; IMC, tabaquismo (paquetes-año), actividad física, historia familiar de diabetes tipo II, cáncer, o enfermedad coronaria, consumo de aspirina, dislipemia, postmenopausia; Modelo 3: modelo $2+$ AHEI. *Quintilo de referencia.

Conclusión

El consumo de granos se asocia a una disminución del riesgo de mor-

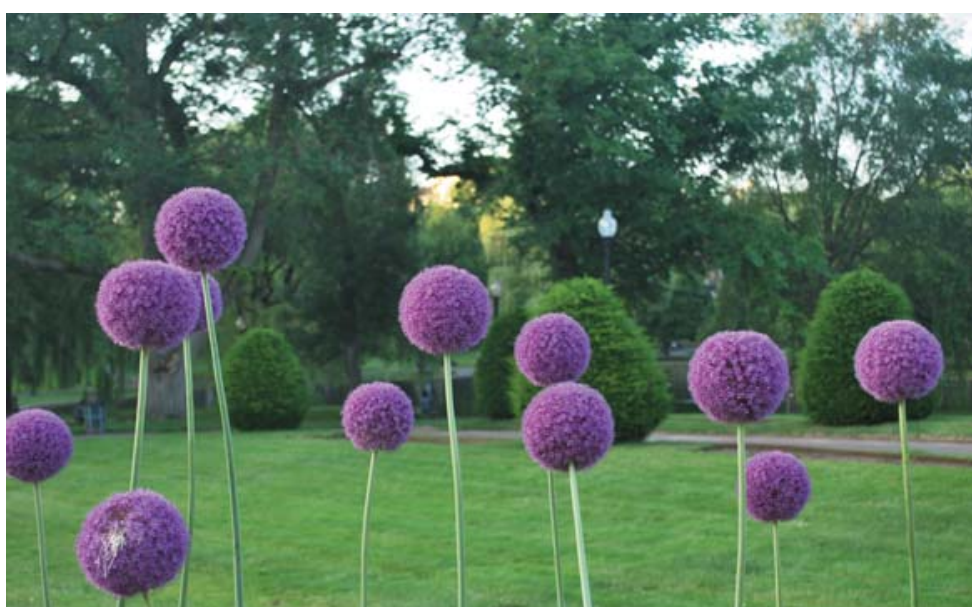




\section{Comentario}

Si bien hay evidencia de que el consumo de granos integrales se encuentra asociado a una disminución en el riesgo de enfermedades crónicas como diabetes tipo $2^{1,2,3}$ y enfermedad cardiovascular ${ }^{2,3,4,}$ poco se sabía sobre cómo, el simple hecho de aumentar la cantidad de granos en la dieta de una persona, puede impactar sobre la mortalidad total y causa-específica. Este trabajo nos provee evidencia de que el aumento de consumo de granos disminuye la mortalidad total y cardiovascular pero no así la mortalidad por cáncer. Esto último probablemente se deba a que se evaluó mortalidad global por cáncer, incluyendo tanto cáncer de colon (para el cual el consumo de granos tiene un efecto protector) ${ }^{5}$, así como también de mama, próstata, ovario y endometrio (para los cuales el consumo de granos no tendría efecto protector) $6,7,8,9,10,11$.

Este trabajo tiene como fortalezas un adecuado ajuste por variables confundidores. Por ejemplo, quienes comen más granos podrían morir menos porque quien come granos tiene además un estilo de vida más saludable (fuma menos, hace más actividad física, tienen menor IMC, etc.). De hecho, esto fue efectivamente es así en el estudio. Sin embargo al ajustarse por estas covariables, nos permitieron apreciar el efecto independiente de la asociación entre consumo de granos y mortalidad.

\section{Conclusiones del comentador}

Frecuentemente recomendamos a nuestros pacientes una dieta saludable, quizás porque lo asociamos con una disminución del riesgo de contraer determinadas enfermedades. Este estudio presenta evidencia robusta sobre el posible impacto beneficioso del consumo de granos sobre la mortalidad.

Granero M. El consumo de granos se asocia a una disminución del riesgo de mortalidad total y por causa cardiovascular. Evid Actual Pract Ambul. 2015;18(4):114-115. Oct-Dic. Comentado de: Wu H, y col. Association Between Dietary Whole Grain Intake and Risk of Mortality: Two Large Prospective Studies in US Men and Women. JAMA Intern Med. 2015;175(3):373-84. PMID: 25559238.

\section{Referencias}

1.Munter JSL, Whole grain, bran, and germ intake and risk of type 2 diabetes: a prospective cohort study and systematic review. PLoS Med. 2007;4(8):e261.

2. Ye EQ, Greater whole-grain intake is associated with lower risk of type 2 diabetes, cardiovascular disease, and weight gain. J Nutr. 2012;142(7):1304-1313.

3. Cho SS, Consumption of cereal fiber, mixtures of whole grains and bran, and whole grains and risk reduction in type 2 diabetes, obesity, and cardiovascular disease. Am J Clin Nutr. 2013;98(2):594619.

4. Mellen PB. Whole grain intake and cardiovascular disease: a meta-analysis. Nutr Metab Cardiovasc Dis. 2008;18(4):283-290.

5. Aune D, et al. Dietary fibre, whole grains, and risk of colorectal cancer: systematic review and dose-response meta-analysis of prospective studies. BMJ. 2011;343:d6617.

6. Aarestrup J, et al. Whole grain, dietary fiber, and incidence of endometrial cancer in a Danish cohort study. Nutr Cancer. 2012;64(8):1160-1168.

7. Kasum CM, lowa Women's Health Study. Whole grain intake and incident endometrial cancer: the lowa Women's Health Study. Nutr Cancer. 2001:39(2):180-186.

8. Hedelin M. Dietary phytoestrogens and the risk of ovarian cancer in the women's lifestyle and health cohort study. Cancer Epidemiol Biomarkers Prev. 2011;20(2):308-317.

9. Nicodemus KK, Whole and refined grain intake and risk of incident postmenopausal breast cancer (United States). Cancer Causes Control. 2001;12(10):917-925.

10. Egeberg R, et al. Intake of whole grain products and risk of breast cancer by hormone receptor status and histology among postmenopausal women. Int J Cancer. 2009;124(3):745-750.

11. Egeberg R, et al. Intake of whole-grain products and risk of prostate cancer among men in the Danish Diet, Cancer and Health cohort study. Cancer Causes Control. 2011;22(8):1133-1139.

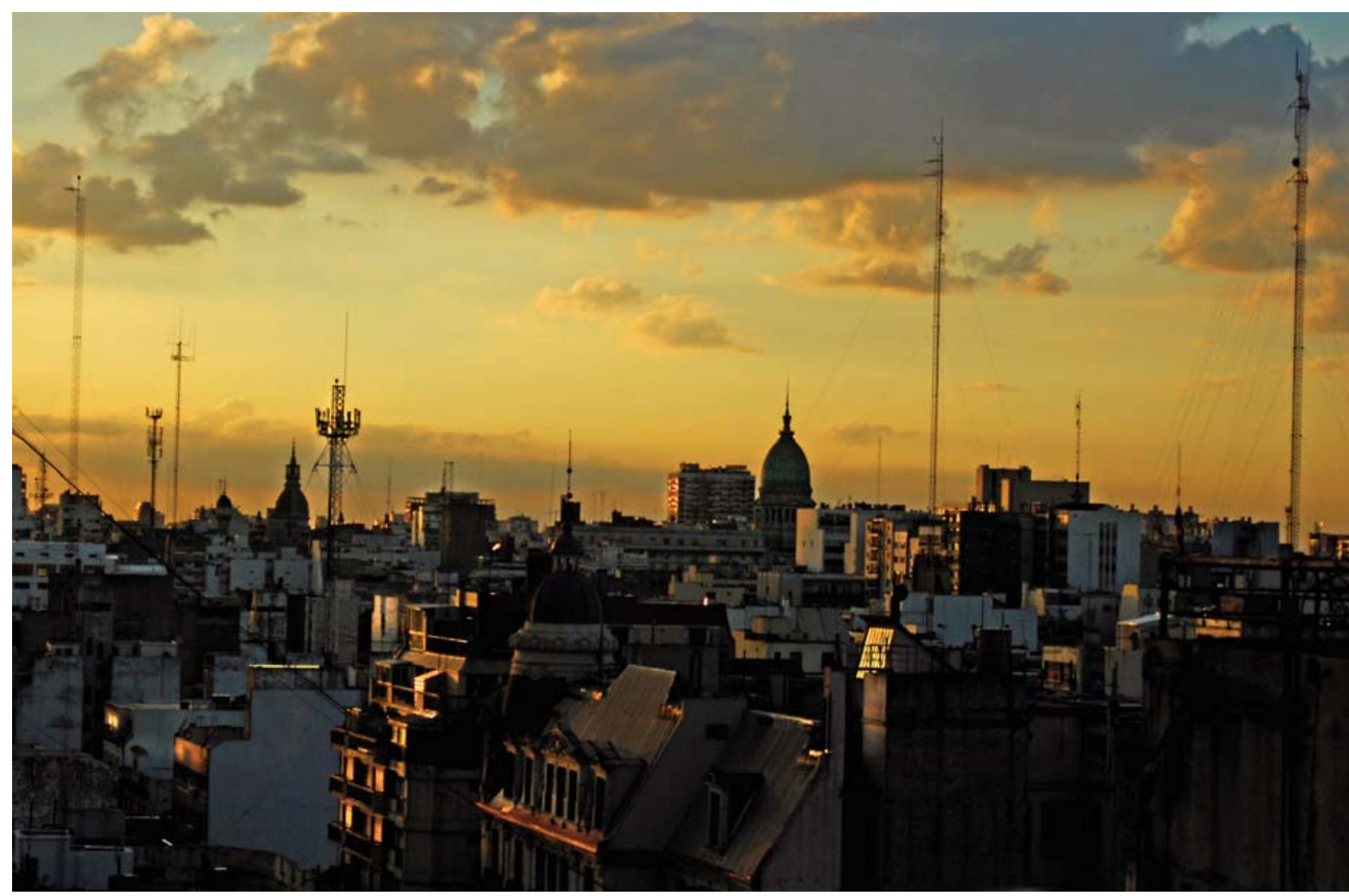

